\title{
Maximum Mass of Neutron Stars with Quark Matter Core
}

\author{
Tatsuyuki Takatsuka*, Tetsuo Hatsuda ${ }^{\dagger, * *}$ and Kota Masuda ${ }^{\dagger}$ \\ *Iwate University, Morioka 020-8550, Japan \\ ${ }^{\dagger}$ Department of Physics, The University of Tokyo, Tokyo 113-0033, Japan \\ ** Theoretical Research Division, Nishina Center, RIKEN, Wako 351-0198, Japan
}

\begin{abstract}
We propose a new strategy to construct the equation of state (EOS) for neutron stars (NSs) with hadron-quark (H-Q) phase transition, by considering three density-regions. We supplement the EOS at H-Q region, very uncertain due to the confinement-deconfinement problems, by sandwitching in between and matching to the relatively" well known" EOSs, i.e., the EOS at lower densities (H-phase up to several times nuclear density, calculated from a G-matrix approach) and that at ultra high densities (Q-phase, form a view of asymptotic freedom). Here, as a first step, we try a simple case and discuss the maximum mass of NSs.
\end{abstract}

Keywords: quark matter, neutron stars, maximum mass

PACS: $26.60 . \mathrm{Kp}, 26.65 . \mathrm{Mn}, 21.65 . \mathrm{Qr}$

\section{INTRODUCTION}

Observations of NS mass $\left(M_{o b s}\right)$ provide a precious probe for the stiffness of the EOS and the composition of NS matter. The larger $M_{o b s}$ demands for the stiffer EOS and thereby gives a stringent condition on the structure since various new phases proposed so far work for the softening of the EOS. For example, the EOS is softened dramatically by a participation of hyperons (Y) in NS cores and the theoretical maximum mass $\left(M_{\text {max }}\right)$ fails to exceed $M_{o b s} \simeq 1.44 M_{\odot}$ for PSR1913+16, suggesting a necessity of extra repulsion in dense hypernuclear systems. It has been shown that this contradiction between theory and observation can be resolved by the introduction of three-body force repulsion (TNR) acting" universally" on $N N, Y N$ and $Y Y$ parts [1].

Recent observation of a very massive NS $\left(M_{o b s}=(1.97 \pm 0.04) M_{\odot}\right)$ for PSR J16142230 [2], inevitably leads to more crucial condition. From a view of the universal TNR, NSs as heavy as $2 M_{\odot}$ are made possible, consistent with the observation [3]. On the other hand, from a view of quark (Q) matter core in NSs, results reported to date are problematic, i.e., $M_{\max } \gtrsim 2 M_{\odot}$ is possible for some works and not for other works. Here we aim to analyze the situation as to the existence of Q-matter core on the basis of a strategy described below.

\section{STRATEGY AND APPROACH}

As illustrated in Fig. 1, our strategy to construct the EOS with hadron-quark (H-Q) phase transition is as follows [4]. The EOS is divided into three parts (I $\sim(\mathrm{III})$ depending on the density $(\rho)$-regions, i.e., the $\operatorname{EOS}(\mathrm{I})$ in pure hadron phase $\left(\rho \lesssim \rho_{H} ; \rho_{H}=\right.$ several 


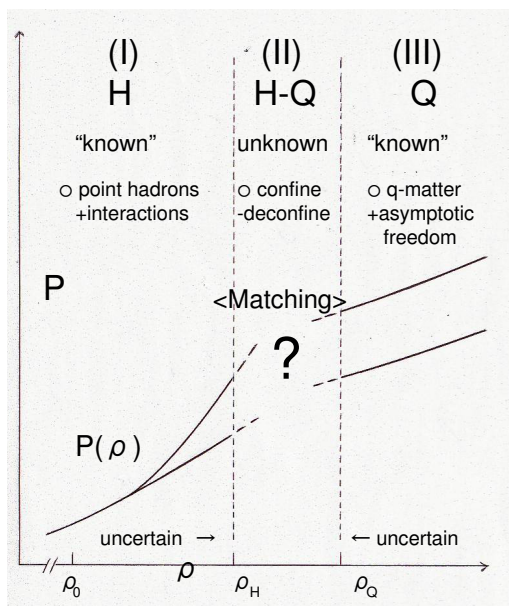

(a)

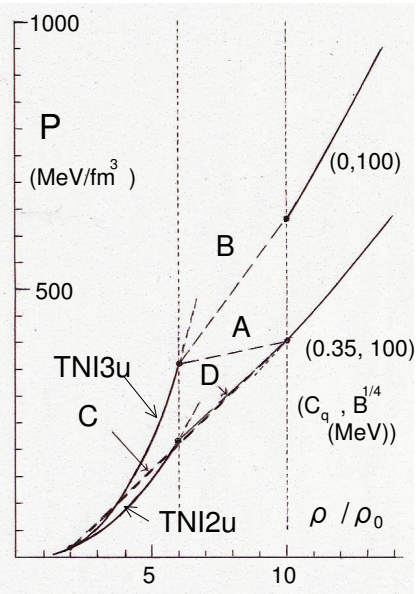

(b)

FIGURE 1. (a) A strategy to construct EOS with hadron -quark phase transition; divided 3 regions and matching. (b) Examples $(A \sim D)$ for linear Interpolation as a first step for matching procedure.

times nuclear density $\rho_{0}, \rho_{0}=0.17$ nucleons $/ \mathrm{fm}^{3}$ ), (II) in hadron-quark transient phase $\left(\rho_{H} \lesssim \rho \lesssim \rho_{Q}\right)$ and (III) in pure quark phase $\left(\rho \gtrsim \rho_{Q} ; \rho_{Q} \sim(8-15) \rho_{0}\right)$. The idea is to supplement very uncertainties of the EOS (II) relevant to confinement-decondinement, by sandwitching (II) in between the relatively" well known" (I) and (II) and matching with (I) at lower densities and (II) at ultra high densities.

In (I), the NS matter is composed of $N(n, p), Y\left(\Lambda, \Sigma^{-}\right)$and leptons $\left(e^{-}, \mu^{-}\right)$and the baryon part EOS is derived by a $G$-matrix-based effective interaction $\tilde{V}$ applicable to $\{N+Y\}$ matter, together with the universal TNR ( $\tilde{U}$; phenomenological one of Illinoi ' s-type, expressed as an effective 2-body force). Parameters in TNR are determined so that the EOS from $\tilde{V}+\tilde{U}$ satisfies the saturation property and symmetry energy of nuclear matter. The stiffness of nuclear-part EOS is specified by the incompressibility $\kappa ; \kappa=300 \mathrm{MeV}(250 \mathrm{MeV})$ for stiffer TNI3u-EOS (softer TNI2u-EOS).

In (III) the NS matter is taken to consist of massless and flavor symmetric $(\{u, d$, $s\}$ )-quark matter and from a viewpoint of asymptotic freedom, the pressure $p$ and the energy density $\varepsilon$ are calculated in a framework of the MIT bag model[5] as

$$
P=\left(1-C_{q}\right) 3 \mu^{4} / 4 \pi^{2}-B_{e f f}, \quad \varepsilon=3 p+4 B_{e f f},
$$

where $C_{q}$ is a correction factor coming from the one-gluon- exchange effects, $\mu=$ $\hbar c\left(\pi^{2} \rho\right)^{1 / 3}$ the chemical potential and $B_{\text {eff }}$ the effective bag constant. Charge neutrality is automatically satisfied by the equal number of $u, d$ and $s\left(\rho_{u}=\rho_{d}=\rho_{s}=\rho\right)$.

\section{SOME RESULTS AND REMARKS}

As a first step for the matching procedure, we try a" linear interpolation" of $p$ and $\varepsilon$ between (I) and (III) as shown in Fig. 2. Results for some cases (A $\sim D$ in Fig. 2) are given in table 1 . We remark the points: (i) $M_{\max }$ of NSs with Q-matter core is controlled predominantly by the EOS in H-phase, i.e., the stiffer the EOS, the larger $M_{\max }$ is, as 
TABLE 1. Some examples for NS models with quark matter core, EOS (II) from simple" linear interpolation"

\begin{tabular}{lrrrrrrrr}
\hline CASE & H-EOS & $C_{q}$ & $B^{1 / 4}$ & $\rho_{H}$ & $\rho_{Q}$ & $M_{\max } / M_{\odot}$ & $\rho_{c} / \rho_{0}$ & $R / \mathrm{km}$ \\
\hline $\mathrm{A}$ & TNI3u & 0.35 & 100 & 6 & 10 & 1.82 & 10.1 & 11.7 \\
$\mathrm{~B}$ & TNI3u & 0. & 100 & 6 & 10 & 1.81 & 10.1 & 8.1 \\
$\mathrm{C}$ & TNI3u & 0.35 & 100 & 2 & 10 & 2.06 & 11.2 & 9.4 \\
$\mathrm{D}$ & TNI2u & 0.35 & 100 & 6 & 10 & 1.62 & 9.0 & 14.8 \\
\hline
\end{tabular}

seen from a comparison of A and D cases. (ii) $M_{\max } \sim 1.8 M_{\odot}$ (A and B) is almost the same as $1.83 M_{\odot}$ (with the central density $\rho_{c} \simeq 8 \rho_{0}$ ) for NSs only with pure H-phase, although with $\rho_{c}$ somewhat shifted to higher density side. So if we adopt more stiffer Hphase EOS, $M_{\max }$ for NSs with Q-matter would exceed $2.0 M_{\odot}$ observed. (iii) The case C with low $\rho_{H}=2 \rho_{0}$ results in $M_{\max } \simeq 2.0 M_{\odot}$ even with the present TNI3u H-phase EOS. In this case, we have unusual aspect for $R-\rho_{c}$ relation, $d R / d \rho_{c}>0$ in contrast with usual $d R / d \rho_{c}<0$, although $d M / d \rho_{c}>0$ as usual. To ask the reason is of great interest in relation to the instability of NS models.

Further investigations to go beyond the present simple approach are now in progress by adopting the superposition of $\mathrm{H}$ - and Q-phase EOSs for matching procedure, using more realistic Q-phase EOS from a chiral NJL model[6] and taking account of a thermodynamic relation between $p$ and $\varepsilon$.

\section{ACKNOWLEDGMENTS}

The authors are grateful to R. Tamagaki for using his computing code of neutron star model and helpful discussions. One of the author (T.T.) thank S. Nishizaki for his collaboration in studies of hyperon-mixed neutron stars. He also thanks T. Tatsumi for his interest in the work.

\section{REFERENCES}

1. T. Takatsuka, Prog. Theor. Phys. Suppl. No. 156, 84 (2004).

2. P. B. Demorest,et.al., Nature, 4671081 (2010).

3. T. Takatsuka, S. Nishizaki and R. Tamagaki, Proc. Int. Symp. " FM50" 209 (AIP Conference proceedings, 2008).

4. T. Takatsuka, Abstract book of JSP meeting, Vol. 66, No. 1, Part I 66 (2011).

5. K. Yagi, T. Hatsuda and Y. Miake," Quark-Gluon Plasma " Chap. 9 (Cambridge Univ. Press, Cambridge, 2008).

6. T. Hatsuda and T. Kunihiro, Phys. Reports 247221 (1994). 